\title{
ALTERATION OF THE GUT MICROBIOME FOR PATIENTS WITH INFLAMMATORY BOWEL DISEASE: A REVIEW
}

\author{
AL-AMraH, H. ${ }^{{ }^{*}}$-SAADAH, O. I. ${ }^{2,3}-$ MOSLI, M. ${ }^{3,4}-$ EDRIS, S. ${ }^{1,5,6}-$ AlHINDI, R. ${ }^{1}-$ \\ BAHIELDIN, A. ${ }^{1,5}$ \\ ${ }^{I}$ Department of Biological Sciences, Faculty of Science, King Abdulaziz University, Jeddah, \\ Saudi Arabia \\ ${ }^{2}$ Department of Paediatrics, Faculty of Medicine, Inflammatory Bowel Disease Research \\ Group, King Abdul-Aziz University, Jeddah, Saudi Arabia \\ ${ }^{3}$ Inflammatory Bowel Disease Research Group, King Abdulaziz University, Jeddah, Saudi \\ Arabia \\ ${ }^{4}$ Department of Medicine, King Abdulaziz University, Jeddah, Saudi Arabia \\ ${ }^{5}$ Department of Genetics, Faculty of Agriculture, Ain Shams University, Cairo, Egypt \\ ${ }^{6}$ Princess Al Jawhara Albrahim Center of Excellence in Research of Hereditary Disorders \\ (PACER-HD), Faculty of Medicine, King Abdulaziz University, Jeddah, Saudi Arabia \\ ${ }^{*}$ Corresponding author \\ e-mail: hggaber@kau.edu.sa; phone: +966-012-460-0000; \\ address: P. O. Box 80200, Jeddah 21589, Saudi Arabia
}

(Received 30 ${ }^{\text {th }}$ May 2020; accepted $20^{\text {th }}$ Aug 2020)

\begin{abstract}
Inflammatory bowel disease (IBD) is a set of multifactorial gut inflammatory conditions. The most common types of IBD are ulcerative colitis (UC) and Crohn's disease (CD), which are attributed to a deregulated immune response to an imbalance in the gut microbiome. The occurrence of IBD is increasing worldwide, with over one million people in the USA and 2.5 million in Europe estimated to have one form of the disease. Furthermore, an increase in IBD has recently been reported in industrialized countries in Asia, South America, Africa and the Middle East, which suggests that it has developed into a global disease with rising prevalence in each continent that may incur substantial healthcare costs in the future. Studying the gut microbiome of patients with IBD can provide a deeper understanding of the role that gut microbiota plays in the development of disease. This will further help in therapeutic microbiome manipulation of patients with IBD.
\end{abstract}

Keywords: ulcerative colitis, Crohn's disease, immune response, intestinal microbiota, dysbiosis

\section{Introduction}

Bacteria, viruses, fungi, protozoa, and many other microorganisms can live together as a community, but can also live in close association with humans, plants, and other complex organisms. This is possible through relationships of parasitism, commensalism, and mutualism between microorganisms and their hosts (Lederberg and McCray, 2001). The interacting community of microorganisms within or in proximity to multicellular organisms is referred to as a microbiome. The microbiome forms an ecological community of pathogenic, commensal, and symbiotic microorganisms in a biological environment, such as the human body. There is a strong mutual relationship between microbes and humans as the human body can benefit from microbe's ability to support the conditions necessary for human healthy life, while microbes benefit from 
obtaining needed resources of the human body. The nasal, skin, oral, urogenital, and gastrointestinal linings of an individual comprise unique and varying microbial communities. The human microbiome is a significant contributor to balanced immunogenicity through symbiosis. Alteration of the microbiome can result in the emergence of diseases. Thus, the condition of microbiomes is an essential effector on human health. Understanding the microbial compositions of healthy individuals is key to determining the influence of the microbiome on the occurrence of human disease.

Inflammatory bowel disease (IBD) and a number of other diseases have exhibited differences in the function and structure of gut microbiota. This can be observed in healthy humans, where changes in gut microbiota can result in a disturbance of the immune system. Over one million Americans and two million Europeans have been reported to suffer from relapsing-remitting forms of IBD (Kaplan, 2015). The data suggests an increase in IBD globally, but with the highest incidence and prevalence reported in Western countries. Nonetheless, the newly industrializing countries of Asia, South America, Africa, and the Middle East, have reported a rapid increase in IBD (Kaplan, 2015).

Lozupone et al. (2012) have characterized the pathophysiology of IBD as a reluctance to regulate immune response to imbalance in the gut microbiome. Therefore, compared to healthy individuals, patients with IBD have lower numbers of bacteria with anti-inflammatory capability and an increased number of bacteria with proinflammatory capability. Dysbiosis is currently considered the most appealing target for scientific research on IBD as it gives the opportunity for clinicians to intervene and alter the natural course of the disease. Given the large variation in gut microbiota across populations, the complex task of mapping the microbiota of healthy populations must be carried out. The microbiota of the diseased population was recently mapped though the Human Microbiome Project (HMP) run by NIH.

\section{The Human Gut Microbiome}

There are about a hundred trillion microbial organisms that comprise the human gut microbiota (Lozupone et al., 2012). These diverse organisms include viruses, protozoa, fungi and bacteria, which comprise more than 1000 different types of bacterial species (Honda and Littman, 2012) (Fig. 1). These bacterial species are made up of more than three million non-redundant microbial genes (Qin et al., 2010). A number of factors, including diet (Collado et al., 2010; Goldsmith and Sartor, 2014), age (Hopkins et al., 2001), gender, genetic composition (Khachatryan et al., 2008), geographic location (Sonnenburg et al., 2004), and health or disease status (Collado et al., 2010) of the individual influence the gut microbiota after birth. Four phyla (e.g., Bacteroidetes, Actinobacteria, Proteobacteria, Firmicutes) were found to dominate over $99 \%$ of human intestinal bacteria (Ley et al., 2008), of which two phyla (Firmicutes and Bacteroidetes) are most common in the intestinal bacteriome of healthy adults (Andoh, 2016). These microbes are densely populated in the colon and the distal ileum (Eckburg et al., 2005). The human gut hosts a large microbial community whose genetic content is described as a metagenome. The colonizers consist of a metagenome made up of 100 times the population of genes in the human genome. Dominguez-Bello et al. (2010) indicated that this metagenome is usually investigated by targeted sequencing of marker genes, including $16 \mathrm{~S}$ ribosomal RNA. 


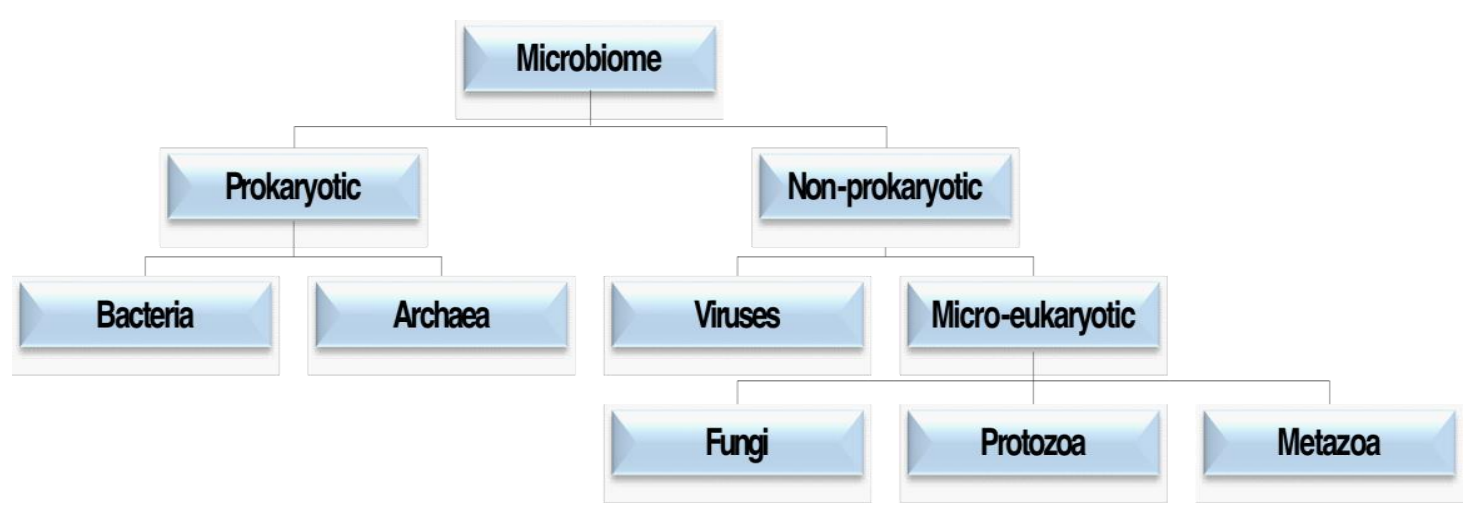

Figure 1. Microorganisms of the human gut microbiome

\section{Development Process of the Human Microbiota}

As a person ages, the microbiota varies in stability and diversity. Interactions between the gut microbiome and the host begin from birth and continue throughout life. In the early years of an individual, the density and stability of the microbiome are usually low (Claesson et al., 2011). By early adulthood, the microbiome begins to demonstrate significant diversity and stability (Lozupone et al., 2012), while the microbiome reaches maximum complexity and remains relatively stable throughout most of an adult's life. However, in later years and in the elderly, Biagi et al. (2010) note that the stability and diversity of the microbiome decreases.

The mode of birth highly determines the microbiota of an infant. In the case of vaginal birth, the microbiota of the infant takes after that of the mother. In a Caesarian birth, as Dominguez-Bello et al. (2010) pointed out that the microbiota in the skin of the newborn takes the characteristics of the mother. Feeding methods also affect the establishment of the microbiome; the microbiota of formula- and breast-fed newborns differ both in diversity and structure (Guaraldi and Salvatori, 2012). While the microbiota of breast-fed babies basically comprises Bifidobacteria, Penders et al. (2006) noted that microbiota of bottle-fed with formula babies basically consists of Clostridium difficile, lactobacilli predominate, Bacteroides fragilis, and Escherichia coli. At the age of three, a child's microbiome resembles that of an adult, and stabilizes with time. At this age, the number of species approximates 100, although this continues to increase and may exceed 1000 in adulthood. The ecological system of the gut also stabilizes and becomes more complex in adult life owing to the predominant composition of Firmicutes and Bacteroidetes (Fig. 2). However, old age causes a reduction in the stability of the ecosystem. Notably, the dominating phyla at this stage shifts from Firmicutes to Bacteroides, and the number of Bifidobacteria reduces while that of Proteobacteria rises. As an individual reaches old age, the microbiota shifts towards a Clostridium- dominated community, and the population of Bacteroidetes rises. This explains the significant difference between the microbiota of a young adult and that of an older person (Penders et al., 2006). Pregnancy enhances the development of Proteobacteria and Actinobacteria, as well as enhancing diversity. However, the gut microbiota gradually regains its original formulation after delivery. 


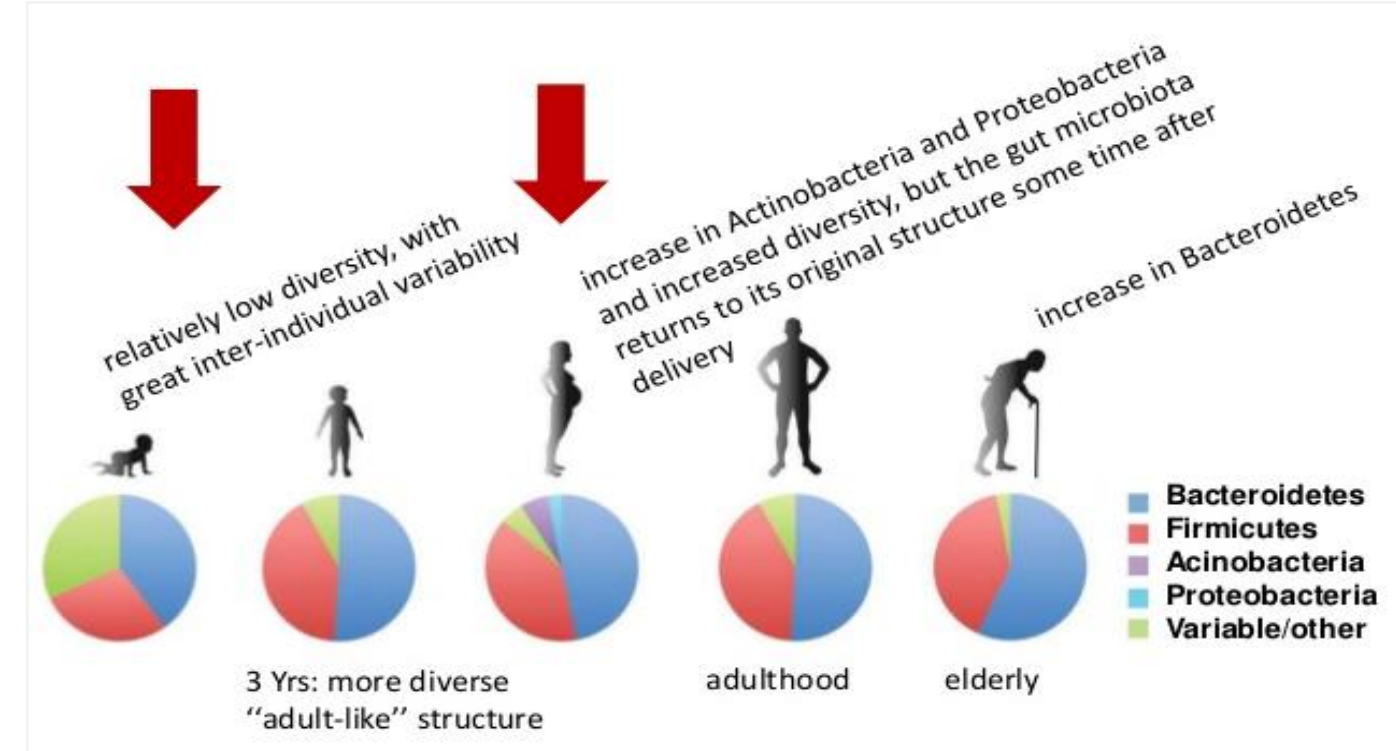

Figure 2. The structure of the human intestinal microbiota across the life cycle. Modified from Kostic et al. (2013)

\section{Effects of Geology on Gut Microbial Assorted Variety and Stability}

A geographical region can be associated with various ways of life that correspond with a variety of dietary practices (including such factors as country, city, countryside, religion, etc.). In the microbiota creation in volunteers from Venezuela, Malawi, and the US (0-70 years old), it was found that regardless of age, the microbiota structure could be categorized by country (Yatsunenko et al., 2012). The least microbially diverse group in that investigation was grown-up Americans of which the bacteria Prevotella, in particular, was underrepresented in this group. When comparing African and European children, De Filippo et al. (2010) discovered a greater number of Prevotella in African children. The fecal microbiota of African children is rich in Actinobacteria and Bacteroidetes, but has lower quantities of Firmicutes; European children have microbiota that are rich in Proteobacteria and over double the normal quantity of Firmicutes compared to Bacteroidetes. Moreover, Ou et al. (2013) noted a greater quantity of Prevotella in Africans in contrast to African Americans. A comparative study was carried out between the Tanzanian Hadza hunter-gatherers, and Italians (Schnorr et al., 2014). In a few African populaces in this examination, high levels of Succinivibrio and Treponema exist. These two microscopic organisms have a high fiber-debasing potential. This high potential may be predictable owing to a vigorously plant-based diet. Additionally, the Hadza gut microbial environment has relatively low levels of Bifidobacterium. This is thought to be the effect of the absence of dairy foods in the diet and lack of contact with domesticated animals.

\section{Inflammatory Bowel Disease (IBD)}

IBD is defined as a chronic intestinal inflammatory condition caused by hostmicrobe interactions in genetically susceptible individuals. The most common types of IBD are ulcerative colitis (UC) and Crohn's disease (CD), and the basic distinctions between them are location and severity. CD can affect any part of the digestive tract, but 
UC develops only in the colon and rectum. Patients with IBD often suffer from ongoing symptoms of abdominal pain, diarrhea, gastrointestinal bleeding, and malnutrition. Persistent disease activity has been linked to repeated hospitalizations and development of complications, such as the need for bowel resection, colorectal cancer, and mortality (Fakhoury et al., 2014). Both exogenous factors (abnormal microbiota) and host factors (intestinal epithelial cell barrier function, and innate and adaptive immune function) cause a chronic state of dysregulated mucosal immunity.

\section{Global Incidence and Prevalence of Inflammatory Bowel Disease}

The occurrence of IBD is increasing worldwide in the 21st Century. The prevalence of IBD varies with geographical region. The highest reported prevalence rates of the IBD are in Europe (UC, 505 per 1,000,000 persons/year; CD, 322 per 1,000,000 persons/year) ( $\mathrm{Ng}$ et al., 2017). Regions with the highest annual incidence of IBD burden are Europe (UC, 24.3 per 100,000 persons/year; CD, 12.7 per 100,000 persons/year) and North America (UC, 19.2 per 100,000 persons/year; CD, 20.2 per 100,000 persons/year). The regions with the lowest reported IBD incidence are Asia and the Middle East (UC, 6.3 per 100,000 persons/year; CD, 5.0 per 100,000 persons/year) (Ng et al., 2017).

Urban areas may show a higher prevalence of CD than rural areas, and CD may also be higher in areas of higher socio-economic classes (Soon et al., 2012). The incidence of the disease begins to increase mostly among economically stable individuals. However, the ailment becomes more complex with time. An individual who initially belongs to low-incidence populations and migrates to a developed country before adolescence may show an increased prevalence of IBD. This is often the case with the first generation setting in a nation with increased IBD prevalence.

The "hygiene hypothesis" aims to explain the variation in incidence levels between developing and developed countries. According to this theory, an individual less exposed to childhood infections loses potentially beneficial organisms that enhance the development of a regulatory $\mathrm{T}$ cell. Additionally, such a person may not develop a strong immune repertoire because he or she has not encountered the noxious organisms (Pugazhendhi et al., 2011; Sood et al., 2014). Thus, such an individual might be more likely to develop IBD and other chronic immune diseases. It is often surmised that the emergence of IBD in Third World nations is a result of adoption of a Western lifestyle, including diet and the reliance on Western approaches to vaccination and medication. The significance of such developments in early life may be of particular significance. Though UC has been documented to have emerged before CD, the prevalence of CD in developed nations has overtaken that of UC in the past 20 years. However, developing nations have recorded an increasing incidence level of UC. India, for example, has recorded a drop in $\mathrm{UC} / \mathrm{CD}$ ratio from 10:1 to 8:1. One notable trend is that the prevalence of $\mathrm{CD}$ rises once the disease has been present in a population for some time. Hong Kong has observed a UC/CD ratio that has declined to 1:1 from 8:1 ( $\mathrm{Ng}$ et al., 2015). In general, the incidence of CD reaches its optimum in early adulthood, and the incidence rate declines in the elderly. The incidence of UC remains stable from early adulthood to retirement age.

East Asia and other parts of Asia have reported a trend of rising prevalence and incidence of IBD. Despite this trend being more acute in developing nations, some developed countries, such as Japan, have been shown to be affected. Also, the CD incidence rate has been more pronounced in female adults over male adults, although 
the past decade has recorded a higher incidence of $\mathrm{CD}$ in boys than in girls in developing countries. Over time, it may transpire that an equal CD prevalence for both sexes is reached. For example, studies from East Asia are suggesting a male predominance of $\mathrm{CD}$ and an equal sex ratio of UC ( $\mathrm{Ng}$ et al., 2016).

\section{Alterations in Intestinal Microbiota Implicated in the Development of IBD}

The gut microbiota is believed to play a central role in the pathogenesis of IBD. Many studies give corroborated evidence for gut microbiota dysbiosis in IBD patients compared to healthy individuals. The gut microbiota of healthy people is made principally of microorganisms from the phyla Bacteroidetes (for the most part Bacteroides or Prevotella species), which are gram-negative, or Firmicutes (for the most part Clostridium and Lactobacillus species), which are gram-positive. Actinobacteria (that incorporate Bifidobacterium sp.), Proteobacteria (including Escherichia coli), and Verrucomicrobia (including Akkermansia mucinophila) are commonly present in small numbers in the gut microbiota of healthy individuals. The creation of gut microbiota varies in people with respect to age and advancement of disease (Huttenhower et al., 2012) (Table 1).

Table 1. Composition of intestinal microbiota in healthy humans

\begin{tabular}{|c|c|c|}
\hline Reference & Sample & Microbial signature \\
\hline $\begin{array}{c}\text { (King et al., } \\
\text { 2019) }\end{array}$ & $\begin{array}{c}50 \text { healthy samples } \\
\text { sequenced at GWU and } 49 \\
\text { healthy samples taken from } \\
\text { The Human Microbiome } \\
\text { Project }\end{array}$ & 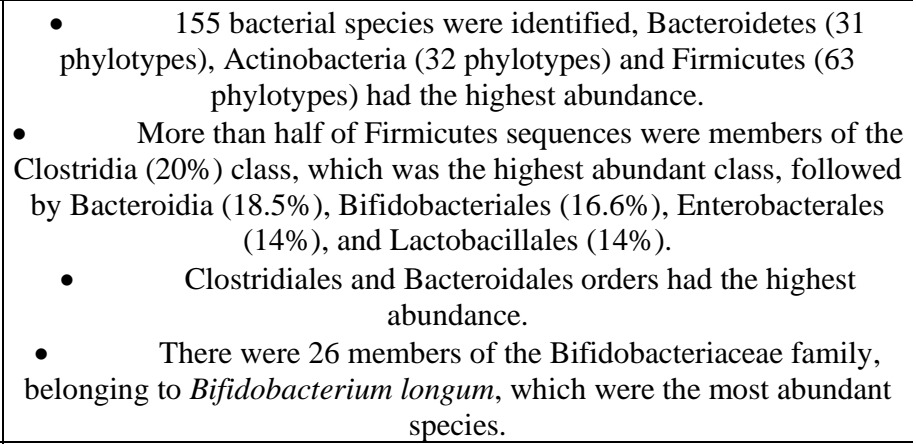 \\
\hline $\begin{array}{l}\text { (Gill et al., } \\
\text { 2006) }\end{array}$ & 2 healthy adult subjects & $\begin{array}{l}133 \text { bacterial phylotypes were identified; Actinobacteria (10 } \\
\text { phylotypes) and Firmicutes (62 phylotypes) had the highest abundance. } \\
60 \% \text { of the Firmicute sequences were members of Clostridia } \\
\text { class (including Clostridia cluster XIV and Faecalibacteria.). }\end{array}$ \\
\hline $\begin{array}{c}\text { (Eckburg et al., } \\
2005)\end{array}$ & 3 healthy adult subjects & $\begin{array}{c}\text { - } 395 \text { bacterial phylotypes were identified, Bacteroidetes (65 } \\
\text { phylotypes) and Firmicutes (301 phylotypes) had the greatest quantities. } \\
\text { Most (95\%) of the Firmicutes sequences were members of the } \\
\text { Clostridia class. } \\
\text { Low abundance of Proteobacteria sequences (including } \\
\text { Escherichia coli), Actinobacteria, Fusobacteria, and Verrucomicrobia } \\
\text { phyla. } \\
\text { Bacteroides thetaiotaomicron were detected in each subject. }\end{array}$ \\
\hline
\end{tabular}

GWU = George Washington University

A change in microflora synthesis, as in IBD, can contribute to intestinal damage. A few examinations have exhibited the Firmicutes phylum were less well represented in BD patients compared to healthy controls (Huttenhower et al., 2012; Rajilić-Stojanović et al., 2013), whereas members of Gammaproteobacteria were relatively light (RajilićStojanović et al., 2013; Sokol et al., 2017) (Table 2). In CD patients, the Clostridia cluster IV group, in particular Faecalibacterium sp., has been shown to relatively low 
(Gill et al., 2006). Members of the Clostridia group XIVa, belonging to the Roseburia genus, also seem to be low in IBD patients (Rajilić-Stojanović et al., 2013). Data on Bacteroidetes report a general decrease in biodiversity in IBD patients (Mar et al., 2016; Sokol et al., 2017), also, significantly lower levels of putative beneficial OTUs; including Prevotella copri and the butyrate-producing bacterium Faecalibacterium prauznitzii (Willing et al., 2010).

Table 2. Changes in gut bacteriome composition in IBD patients

\begin{tabular}{|c|c|c|c|c|c|}
\hline \multirow{2}{*}{ Reference } & \multirow{2}{*}{ Sample } & \multicolumn{4}{|c|}{ Microbial signature } \\
\hline & & Phylum & Class & Family & Genus \& Species \\
\hline \multirow{10}{*}{$\begin{array}{l}\text { Sokol et al. } \\
\text { (2017) }\end{array}$} & \multirow{10}{*}{$\begin{array}{c}\text { Adult (235 } \\
\text { patients with } \\
\text { IBD, } 38 \\
\text { control) fecal } \\
\text { sample. }\end{array}$} & \multirow{2}{*}{$\downarrow$ Bacteroidetes } & \multirow{2}{*}{ Bacteroidia } & $\downarrow$ Bacteroidaceae & ------- \\
\hline & & & & $\downarrow$ Prevotellaceae & $\begin{array}{l}------ \\
\end{array}$ \\
\hline & & \multirow{5}{*}{$\downarrow$ Firmicutes } & \multirow{2}{*}{ Bacilli } & $\uparrow$ Streptococcaceae & ------- \\
\hline & & & & $\uparrow$ Erysipelotrichaceae & ------- \\
\hline & & & \multirow{2}{*}{ Clostridia } & $\downarrow$ Lachnospiraceae & ------ \\
\hline & & & & $\downarrow$ Ruminococcaceae & +----- \\
\hline & & & Negativicutes & $\downarrow$ Veillonellaceae & ------- \\
\hline & & $\uparrow$ Proteobacteria & Gamma & $\uparrow$ Pseudomonadaceae & ------- \\
\hline & & |riotevodienta & proteobacteria & $\uparrow$ Enterobacteriaceae & ------- \\
\hline & & $\uparrow$ Fusobacteria & Fusobacteria & $\uparrow$ Fusobacteriaceae & ------- \\
\hline \multirow{9}{*}{$\begin{array}{l}\text { Eun et al. } \\
\text { (2016) }\end{array}$} & \multirow{9}{*}{$\begin{array}{c}\text { Adult (15 } \\
\text { CD, } 15 \\
\text { control), } \\
\text { fecal sample }\end{array}$} & \multirow{5}{*}{$\downarrow$ Bacteroidetes } & \multirow{2}{*}{ Bacteroidia } & $\downarrow$ Bacteroidaceae & ------- \\
\hline & & & & $\downarrow$ Prevotellaceae & ------- \\
\hline & & & \multirow{2}{*}{ Clostridia } & $\downarrow$ Lachnospiraceae & ------- \\
\hline & & & & $\downarrow$ Ruminococcaceae & ------- \\
\hline & & & Negativicutes & $\downarrow$ Veillonellaceae & $\begin{array}{ll}------ \\
\end{array}$ \\
\hline & & \multirow{3}{*}{$\uparrow$ Proteobacteria } & \multirow{3}{*}{$\begin{array}{c}\text { Gamma } \\
\text { proteobacteria }\end{array}$} & Pseudomonadaceae & $\uparrow$ Pseudomonas sp. \\
\hline & & & & \multirow{2}{*}{ Enterobacteriaceae } & $\uparrow$ Escherichia sp. \\
\hline & & & & & $\uparrow$ Shigella sp. \\
\hline & & $\uparrow$ Fusobacteria & Fusobacteria & Fusobacteriaceae & $\uparrow$ Fusobacterium sp. \\
\hline \multirow{7}{*}{$\begin{array}{l}\text { Mar et al. } \\
\text { (2016) }\end{array}$} & \multirow{7}{*}{$\begin{array}{c}\text { Adult (30 } \\
\text { UC, } 13 \\
\text { control), } \\
\text { fecal sample }\end{array}$} & \multirow{2}{*}{$\downarrow$ Bacteroidetes } & \multirow{2}{*}{ Bacteroidia } & Prevotellaceae & $\downarrow$ Prevotella sp. \\
\hline & & & & Bacteroidaceae & $\downarrow$ Bacteroides sp. \\
\hline & & \multirow{4}{*}{$\downarrow$ Firmicutes } & \multirow{2}{*}{ Clostridia } & Lachnospiraceae & \multirow{2}{*}{$\begin{array}{c}\downarrow \text { A number of } \\
\text { unclassified species }\end{array}$} \\
\hline & & & & Ruminococcaceae & \\
\hline & & & $3 a$ & Streptococcaceae & $\uparrow$ Streptococcus sp. \\
\hline & & & Bacmill & Enterococcaceae & $\uparrow$ Enterococcus sp. \\
\hline & & $\uparrow$ Actinobacteria & Actinobacteria & Bifidobacteriaceae & $\uparrow$ Bifidobacterium sp. \\
\hline & & & & Ruminococcaceae & $\downarrow$ Subdoligranulum $s p$. \\
\hline & & & & Kummococcacede & $\downarrow$ Feacalibacterium $s p$ \\
\hline Quince et al. & $\begin{array}{l}23 \mathrm{CD}, 21 \\
\text { control), }\end{array}$ & Firmicutes & Clostridia & Peptostreptococcaceae & $\begin{array}{c}\uparrow \text { Peptostreptococcus } \\
\text { sp. }\end{array}$ \\
\hline & fecal sample & & & $\downarrow$ Lachnospiraceae & ------ \\
\hline & & & Bacilli & $\uparrow$ Enterococcaceae & ------- \\
\hline & & Actinobacteria & Actinobacteria & Coriobacteriaceae & $\uparrow$ Atopobium sp. \\
\hline & & & & Ruminoccaceae & $\downarrow$ Ruminococcus bromii \\
\hline & & & & Eubacteriaceae & $\downarrow$ Eubacterium rectale \\
\hline & & Firmicutes & Clostridia & lachnospiraceae & $\downarrow$ Roseburia sp. \\
\hline Rajilić- & $\begin{array}{l}\text { Adult (15 } \\
\text { UC } 15\end{array}$ & & & aceae & $\uparrow$ Peptostreptococcus \\
\hline Stojanović et & control), & & & осcасеае & $\uparrow$ Clostridium difficile \\
\hline al. (2013) & fecal sample & Verrucomicrobia & Verrucomicrobiae & Verrucomicrobiaceae & $\downarrow$ Akkermansia $s p$. \\
\hline & & Fusobacteria & Fusobacteria & Fusobacteriaceae & $\uparrow$ Fusobacterium sp. \\
\hline & & & Epsilonproteobac- & Helicobacteraceae & $\uparrow$ Helibacter sp. \\
\hline & & a & teria & Campylbacteraceae & $\uparrow$ Campylobacter sp. \\
\hline
\end{tabular}

IBD, Inflammatory bowel disease; CD, Crohn's disease; UC, Ulcerative colitis; $\uparrow$, Increased; $\downarrow$, Decrease 


\section{The Physiological Functions of the Gut Microbiota}

The physiological benefits that gut microbiota have on the host are: 1) to supply of nutrients and energy (sustenance), 2) improvement of the immune system, and 3) functioning as host gatekeeper (Fig. 3) (Nishida et al., 2018). Details of these benefits are the following.

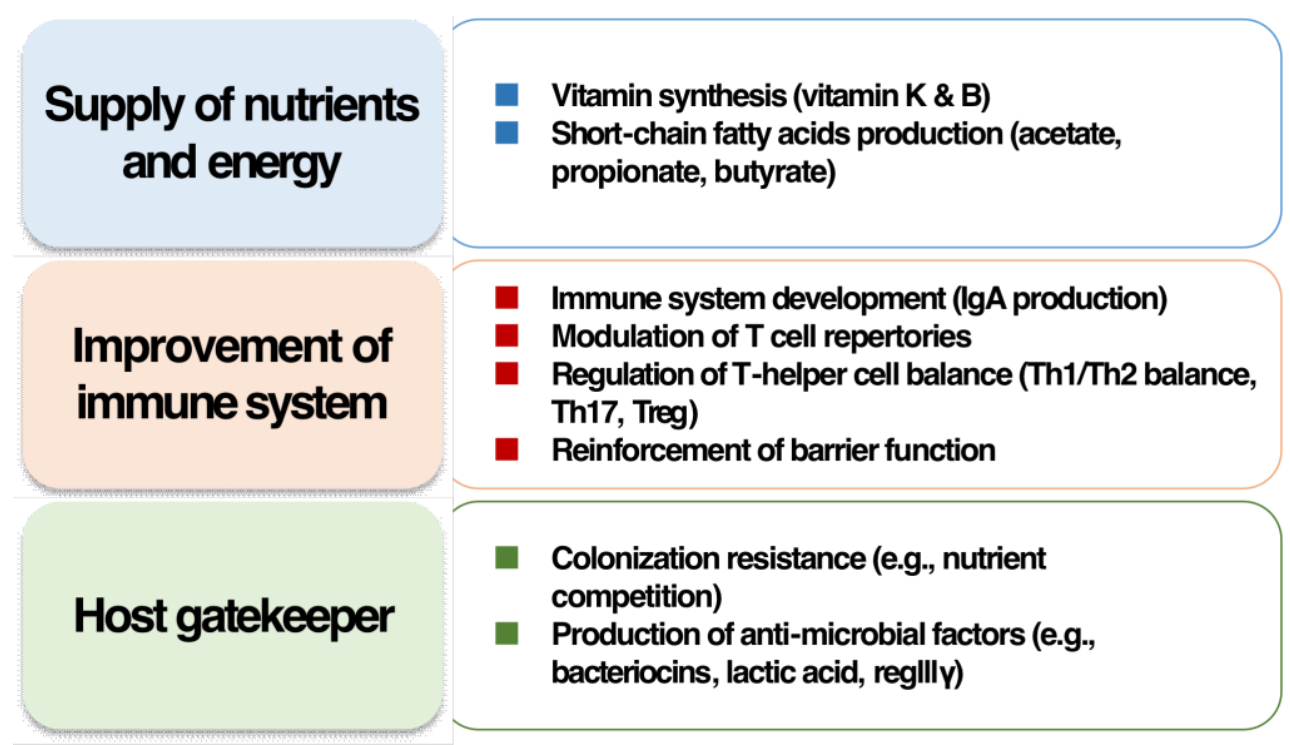

Figure 3. Physiological functions of gut microbiota. Modified from Nishida et al. (2018)

\section{Supply of Nutrients and Energy}

The gut microbiota supply energy and nutrients to the host (O'Hara and Shanahan, 2006). Commensal microorganisms in humans, for instance Bifidobacterium, can coordinate and provide supplements including water-dissolvable B vitamins and vitamin $\mathrm{K}$ (LeBlanc et al., 2011). In addition, intestinal bacteria provide short-chain fatty acids (SCFAs; C2-C6) by fermenting resistant starch or indigestible carbohydrates (dietary fiber). The Bacteroidetes and Firmicutes produce SCFAs from indigestible carbohydrates via participation together with species that have some ability to ferment oligosaccharide (e.g., Bifidobacterial). Anions in the colon are SCFAs, overwhelmingly, acetate, butyrate, and propionate (Marchesi et al., 2016). The latter are vital for a number of aspects of the host's physiology. For example, they are important for supplementing procurement, immune capacity, cell signaling, expansion control, and pathogen protection. SCFA levels are important for their anti-inflammatory effects and imperative for the upkeep of the mucosal barrier; for example, butyrate positively affects cell multiplication, separation, and development after epithelial damage (Lopetuso et al., 2016). The levels of SCFAs are significantly decreased in IBD, which may be a key factor in the degeneration of intestinal and immune homeostasis. The SCFA-producing bacteria are decreased in IBD patients, which include Faecalibacterium (Frank et al., 2007), Odoribacter, Leuconostocaceae, Phascolarctobacterium, and Roseburia (Ahuja, 2015). 


\section{Immune System Improvement}

Microbiota that live in the intestinal tract play a vital role in the improvement of the host immune system. The host immune system, in turn, shapes the structure and function of gut microbiota (Kamada and Núñez, 2014). Germ-free (GF) mice (insufficient in the gut microbiota) show impaired immune development, which is distinguished by immature lymphoid tissues (Bouskra et al., 2008), reduced amounts of intestinal lymphocytes, and diminished elements of antimicrobial peptides (Cash et al., 2006) and immunoglobulin A (IgA) (Hapfelmeier et al., 2010). Reconstitution of the gut microbiota of mice is sufficient to restore these abnormalities of the immune system (Umesaki et al., 1995). One of these microorganisms is Candidatus Arthromitis, known as a segmented filamentous bacteria (SFB). The colonization of SFB alone advances the improvement of the mucosal immune system (Ivanov et al., 2009). The improvement of the host immune system is dependent on host-unequivocal microbiota, as the immune system becomes underdeveloped in GF mice colonized with human microbiota. The gut microbiota, furthermore, regulates $\mathrm{T}$-cell repertoires and controls the $\mathrm{T}$ helper (Th) cell profile (Shanahan, 2002). Authoritative white platelets (in like manner called Tregs) are CD4+ lymphocytes, which coordinate or smother distinctive cells in the immune system (Littman and Rudensky, 2010). It has been shown that SCFA-producing strain in Clostridium clusters IV, XIVa, and XVIII from a sound human fecal precedent started the partition and improvement of colonic Tregs through butyrate production (Atarashi et al., 2013). This result supports part of the clinical data. The degree of Clostridium bunches XIVa and IV in the fecal models is lower in IBD patients compared to healthy individuals (Frank et al., 2007). The low number of Faecalibacterium prausnitzii, which belong to Clostridium cluster IV, is related to a high risk of recurrence of CD after surgery (Walker et al., 2011). The headway of Th17 cells, which are a subpopulation of effector T cells, is defined by their production of interleukin (IL)-17A, IL-17F, IL-21, and IL-22 (Littman and Rudensky, 2010), that are modulated by the gut microbiota. In GF or antibiotic-treated mice, the abundance of Th17 cells in the intestinal mucosa is decreased. One study has shown that the gut microbiota plays a part in the improvement of Th17 cells (Wu et al., 2016). A past report has demonstrated that microbes with adhesive properties to intestinal epithelial cells, for instance, Citrobacter rodentium and Escherichia coli (EHEC) O157, advance the enrollment of Th17 cells (Atarashi et al., 2015).

\section{Host Gatekeeper}

The gut microbiota also combats pathogens. Animals in GF condition are powerless to sullying by intestinal pathogens. An anomaly in the mucosal immune system may add to this lack of protection. Another instrument against pathogens that deform the physical and dietary of the gastrointestinal tract is the colonization of commensal microbiota, which neutralizes the colonization of pathogens (O'Hara and Shanahan, 2006; Sekirov et al., 2010). This framework that actively hinders the interruption of pathogens by commensal microorganisms is called a 'colonization obstacle' (Buffie and Pamer, 2013). The gut microbiota improves colonization assurance from intestinal pathogens by direct and indirect mechanisms of action. Some commensal microorganisms truly stifle intestinal pathogens by competing for nutrients or by starting the production of inhibitory substances. Bacteroides thetaiotaomicron, which is an abundant colonic anaerobe, utilizes starch used by Citrobacter rodentium, which 
adds to the dismissal of pathogens from the intestinal lumen (Kamada et al., 2012). B. thuringiensis secretes bacteriocins that target spore-molding Bacilli and Clostridia, for instance, Clostridium difficile (Huang et al., 2016).

Commensal microbiota and microbial products protect against pathogens indirectly by activating immune responses. For instance, lipopolysaccharides and flagellin derived from the gut microbiota enhance the expression of antimicrobial peptide and RegIII $\gamma$, from epithelial cells by stimulating Toll-like receptor $4^{+}$stromal cells and $\mathrm{TLR}^{+} \mathrm{CD} 103^{+}$dendritic cells (Brandl et al., 2008; Kinnebrew et al., 2010). Segmented filamentous bacteria (SFB) promote the secretion of IgA from B cells, the production of antimicrobial peptides, and the development of Th17 cells in the intestinal mucosa (Talham et al., 1999; Ivanov et al., 2009). The gut microbiota plays a central role in the pathogenesis of IBD. A number of studies provide varied evidence for gut microbiota dysbiosis in IBD patients diverging from healthy controls (Sartor and $\mathrm{Wu}, 2017$; Nishino et al., 2018).

\section{Conclusion}

The population of useful bacteria is reduced in IBD, while the population of pathogenetic bacteria appear to grow. While microbiota differ at the phylogenetic level, the presence of inflammation is considered the major engine for microbiome changes in such diseases. Studies provide the platform for investigating microbiome diversity perturbations in diseases with varying etiology as well as plan for therapeutic microbiome manipulation, in populations which are unique, displaying a variable number of enteric infections and gut autoimmune diseases.

\section{REFERENCES}

[1] Ahuja, V. (2015): Inventory of a reservoir: friends \& foes. - The Indian journal of medical research 142: 4.

[2] Andoh, A. (2016): Physiological role of gut microbiota for maintaining human health. Digestion 93: 176-181.

[3] Atarashi, K., Tanoue, T., Oshima, K., Suda, W., Nagano, Y., Nishikawa, H., Fukuda, S., Saito, T., Narushima, S., Hase, K. (2013): T reg induction by a rationally selected mixture of Clostridia strains from the human microbiota. - Nature 500: 232.

[4] Atarashi, K., Tanoue, T., Ando, M., Kamada, N., Nagano, Y., Narushima, S., Suda, W., Imaoka, A., Setoyama, H., Nagamori, T. (2015): Th17 cell induction by adhesion of microbes to intestinal epithelial cells. - Cell 163: 367-380.

[5] Biagi, E., Nylund, L., Candela, M., Ostan, R., Bucci, L., Pini, E., Nikkila, J., Monti, D., Satokari, R., Franceschi, C. (2010): Through ageing, and beyond: gut microbiota and inflammatory status in seniors and centenarians. - PloS one 5: e10667.

[6] Bouskra, D., Brézillon, C., Bérard, M., Werts, C., Varona, R., Boneca, I. G., Eberl, G. (2008): Lymphoid tissue genesis induced by commensals through NOD1 regulates intestinal homeostasis. - Nature 456: 507.

[7] Brandl, K., Plitas, G., Mihu, C. N., Ubeda, C., Jia, T., Fleisher, M., Schnabl, B., DeMatteo, R. P., Pamer, E. G. (2008): Vancomycin-resistant enterococci exploit antibiotic-induced innate immune deficits. - Nature 455: 804.

[8] Buffie, C. G., Pamer, E. G. (2013): Microbiota-mediated colonization resistance against intestinal pathogens. - Nature Reviews Immunology 13: 790. 
[9] Cash, H. L., Whitham, C. V., Behrendt, C. L., Hooper, L. V. (2006): Symbiotic bacteria direct expression of an intestinal bactericidal lectin. - Science 313: 1126-1130.

[10] Claesson, M. J., Cusack, S., O'Sullivan, O., Greene-Diniz, R., de Weerd, H., Flannery, E., Marchesi, J. R., Falush, D., Dinan, T., Fitzgerald, G. (2011): Composition, variability, and temporal stability of the intestinal microbiota of the elderly. - Proceedings of the National Academy of Sciences 108: 4586-4591.

[11] Collado, M. C., Isolauri, E., Laitinen, K., Salminen, S. (2010): Effect of mother's weight on infant's microbiota acquisition, composition, and activity during early infancy: a prospective follow-up study initiated in early pregnancy. - The American journal of clinical nutrition 92: 1023-1030.

[12] De Filippo, C., Cavalieri, D., Di Paola, M., Ramazzotti, M., Poullet, J. B., Massart, S., Collini, S., Pieraccini, G., Lionetti, P. (2010): Impact of diet in shaping gut microbiota revealed by a comparative study in children from Europe and rural Africa. - Proceedings of the National Academy of Sciences 107: 14691-14696.

[13] Dominguez-Bello, M. G., Costello, E. K., Contreras, M., Magris, M., Hidalgo, G., Fierer, N., Knight, R. (2010): Delivery mode shapes the acquisition and structure of the initial microbiota across multiple body habitats in newborns. - Proceedings of the National Academy of Sciences 107: 11971-11975.

[14] Eckburg, P. B., Bik, E. M., Bernstein, C. N., Purdom, E., Dethlefsen, L., Sargent, M., Gill, S. R., Nelson, K. E., Relman, D. A. (2005): Diversity of the human intestinal microbial flora. - Science 308: 1635-1638.

[15] Eun, C. S., Kwak, M.-J., Han, D. S., Lee, A. R., Park, D. I., Yang, S.-K., Kim, Y. S., Kim, J. F. (2016): Does the intestinal microbial community of Korean Crohn's disease patients differ from that of western patients? - BMC Gastroenterology 16: 28.

[16] Fakhoury, M., Coussa-Charley, M., Al-Salami, H., Kahouli, I., Prakash, S. (2014): Use of artificial cell microcapsule containing thalidomide for treating TNBS-induced Crohn's disease in mice. - Current drug delivery 11: 146-153.

[17] Frank, D. N., Amand, A. L. S., Feldman, R. A., Boedeker, E. C., Harpaz, N., Pace, N. R. (2007): Molecular-phylogenetic characterization of microbial community imbalances in human inflammatory bowel diseases. - Proceedings of the National Academy of Sciences 104: 13780-13785.

[18] Gill, S. R., Pop, M., DeBoy, R. T., Eckburg, P. B., Turnbaugh, P. J., Samuel, B. S., Gordon, J. I., Relman, D. A., Fraser-Liggett, C. M., Nelson, K. E. (2006): Metagenomic analysis of the human distal gut microbiome. - Science 312: 1355-1359.

[19] Goldsmith, J. R., Sartor, R. B. (2014): The role of diet on intestinal microbiota metabolism: downstream impacts on host immune function and health, and therapeutic implications. - Journal of gastroenterology 49: 785-798.

[20] Guaraldi, F., Salvatori, G. (2012): Effect of Breast and Formula Feeding on Gut Microbiota Shaping in Newborns. - Frontiers in cellular and infection microbiology 2: 94.

[21] Hapfelmeier, S., Lawson, M. A., Slack, E., Kirundi, J. K., Stoel, M., Heikenwalder, M., Cahenzli, J., Velykoredko, Y., Balmer, M. L., Endt, K. (2010): Reversible microbial colonization of germ-free mice reveals the dynamics of IgA immune responses. - Science 328: 1705-1709.

[22] Honda, K., Littman, D. R. (2012): The microbiome in infectious disease and inflammation. - Annual review of immunology 30: 759-795.

[23] Hopkins, M., Sharp, R., Macfarlane, G. (2001): Age and disease related changes in intestinal bacterial populations assessed by cell culture, 16S rRNA abundance, and community cellular fatty acid profiles. - Gut 48: 198-205.

[24] Huang, T., Zhang, X., Pan, J., Su, X., Jin, X., Guan, X. (2016): Purification and characterization of a novel cold shock protein-like bacteriocin synthesized by Bacillus thuringiensis. - Scientific reports 6: 35560 . 
[25] Huttenhower, C., Gevers, D., Knight, R., Abubucker, S., Badger, J. H., Chinwalla, A. T., Creasy, H. H., Earl, A. M., FitzGerald, M. G., Fulton, R. S. (2012): Structure, function and diversity of the healthy human microbiome. - Nature 486: 207.

[26] Ivanov, I. I., Atarashi, K., Manel, N., Brodie, E. L., Shima, T., Karaoz, U., Wei, D., Goldfarb, K. C., Santee, C. A., Lynch, S. V. (2009): Induction of intestinal Th17 cells by segmented filamentous bacteria. - Cell 139: 485-498.

[27] Kamada, N., Kim, Y.-G., Sham, H. P., Vallance, B. A., Puente, J. L., Martens, E. C., Núñez, G. (2012): Regulated virulence controls the ability of a pathogen to compete with the gut microbiota. - Science 336: 1325-1329.

[28] Kamada, N., Núñez, G. (2014): Regulation of the immune system by the resident intestinal bacteria. - Gastroenterology 146: 1477-1488.

[29] Kaplan, G. G. (2015): The global burden of IBD: from 2015 to 2025. - Nature reviews Gastroenterology and hepatology 12: 720.

[30] Khachatryan, Z. A., Ktsoyan, Z. A., Manukyan, G. P., Kelly, D., Ghazaryan, K. A., Aminov, R. I. (2008): Predominant role of host genetics in controlling the composition of gut microbiota. - PloS one 3: e3064.

[31] King, C. H., Desai, H., Sylvetsky, A. C., LoTempio, J., Ayanyan, S., Carrie, J., Crandall, K. A., Fochtman, B. C., Gasparyan, L., Gulzar, N. (2019): Baseline human gut microbiota profile in healthy people and standard reporting template. - PloS one 14(9): e0206484.

[32] Kinnebrew, M. A., Ubeda, C., Zenewicz, L. A., Smith, N., Flavell, R. A., Pamer, E. G. (2010): Bacterial flagellin stimulates toll-like receptor 5-dependent defense against vancomycin-resistant Enterococcus infection. - The Journal of infectious diseases 201: 534-543.

[33] Kostic, A. D., Howitt, M. R., Garrett, W. S. (2013): Exploring host-microbiota interactions in animal models and humans. - Genes and development 27: 701-718.

[34] LeBlanc, J., Laiño, J. E., del Valle, M. J., Vannini, V., van Sinderen, D., Taranto, M. P., de Valdez, G. F., de Giori, G. S., Sesma, F. (2011): B-Group vitamin production by lactic acid bacteria-current knowledge and potential applications. - Journal of applied microbiology 111: 1297-1309.

[35] Lederberg, J., McCray, A. T. (2001): Ome SweetOmics--A genealogical treasury of words. - The Scientist 15: 8-8.

[36] Ley, R. E., Hamady, M., Lozupone, C., Turnbaugh, P. J., Ramey, R. R., Bircher, J. S., Schlegel, M. L., Tucker, T. A., Schrenzel, M. D., Knight, R. (2008): Evolution of mammals and their gut microbes. - Science 320: 1647-1651.

[37] Littman, D. R., Rudensky, A. Y. (2010): Th17 and regulatory T cells in mediating and restraining inflammation. - Cell 140: 845-858.

[38] Lopetuso, L. R., Scaldaferri, F., Franceschi, F., Gasbarrini, A. (2016): Bacillus clausii and gut homeostasis: state of the art and future perspectives. - Expert review of gastroenterology and hepatology 10: 943-948.

[39] Lozupone, C. A., Stombaugh, J. I., Gordon, J. I., Jansson, J. K., Knight, R. (2012): Diversity, stability and resilience of the human gut microbiota. - Nature 489: 220.

[40] Mar, J. S., LaMere, B. J., Lin, D. L., Levan, S., Nazareth, M., Mahadevan, U., Lynch, S. V. (2016): Disease severity and immune activity relate to distinct interkingdom gut microbiome states in ethnically distinct ulcerative colitis patients. - MBio 7: e01072-16.

[41] Marchesi, J. R., Adams, D. H., Fava, F., Hermes, G. D., Hirschfield, G. M., Hold, G., Quraishi, M. N., Kinross, J., Smidt, H., Tuohy, K. M. (2016): The gut microbiota and host health: a new clinical frontier. - Gut 65: 330-339.

[42] Ng, S. C., Leung, W. K., Li, M. K., Leung, C. M., Hui, Y. T., Ng, C. K. M., Lo, F. H., Tsang, S., Loo, C. K., Chan, Y. K. (2015): Su1303 Prevalence and Disease Characteristics of Inflammatory Bowel Disease (IBD) in Chinese: Results from a Nationwide Population-Based Registry. - Gastroenterology 148: S-467. 
[43] Ng, W. K., Wong, S. H., Ng, S. C. (2016): Changing epidemiological trends of inflammatory bowel disease in Asia. - Intestinal research 14: 111.

[44] Ng, S. C., Shi, H. Y., Hamidi, N., Underwood, F. E., Tang, W., Benchimol, E. I., Panaccione, R., Ghosh, S., Wu, J. C., Chan, F. K. (2017): Worldwide incidence and prevalence of inflammatory bowel disease in the 21 st century: a systematic review of population-based studies. - The Lancet 390: 2769-2778.

[45] Nishida, A., Inoue, R., Inatomi, O., Bamba, S., Naito, Y., Andoh, A. (2018): Gut microbiota in the pathogenesis of inflammatory bowel disease. - Clinical journal of gastroenterology 11: 1-10.

[46] Nishino, K., Nishida, A., Inoue, R., Kawada, Y., Ohno, M., Sakai, S., Inatomi, O., Bamba, S., Sugimoto, M., Kawahara, M. (2018): Analysis of endoscopic brush samples identified mucosa-associated dysbiosis in inflammatory bowel disease. - Journal of gastroenterology 53: 95-106.

[47] O'Hara, A. M., Shanahan, F. (2006): The gut flora as a forgotten organ. - EMBO reports 7: 688-693.

[48] Ou, J., Carbonero, F., Zoetendal, E. G., DeLany, J. P., Wang, M., Newton, K., Gaskins, H. R., O'Keefe, S. J. (2013): Diet, microbiota, and microbial metabolites in colon cancer risk in rural Africans and African Americans. - The American journal of clinical nutrition 98: 111-120.

[49] Penders, J., Thijs, C., Vink, C., Stelma, F. F., Snijders, B., Kummeling, I., van den Brandt, P. A., Stobberingh, E. E. (2006): Factors influencing the composition of the intestinal microbiota in early infancy. - Pediatrics 118: 511-521.

[50] Pugazhendhi, S., Sahu, M. K., Subramanian, V., Pulimood, A., Ramakrishna, B. S. (2011): Environmental factors associated with Crohn's disease in India. - Indian Journal of Gastroenterology 30: 264-269.

[51] Qin, J., Li, R., Raes, J., Arumugam, M., Burgdorf, K. S., Manichanh, C., Nielsen, T., Pons, N., Levenez, F., Yamada, T. (2010): A human gut microbial gene catalogue established by metagenomic sequencing. - Nature 464: 59 .

[52] Quince, C., Ijaz, U. Z., Loman, N., Eren, A. M., Saulnier, D., Russell, J., Haig, S. J., Calus, S. T., Quick, J., Barclay, A. (2015): Extensive modulation of the fecal metagenome in children with Crohn's disease during exclusive enteral nutrition. - The American journal of gastroenterology 110: 1718.

[53] Rajilić-Stojanović, M., Shanahan, F., Guarner, F., de Vos, W. M. (2013): Phylogenetic analysis of dysbiosis in ulcerative colitis during remission. - Inflammatory bowel diseases 19: 481-488.

[54] Sartor, R. B., Wu, G. D. (2017): Roles for intestinal bacteria, viruses, and fungi in pathogenesis of inflammatory bowel diseases and therapeutic approaches. Gastroenterology 152: 327-339.

[55] Schnorr, S. L., Candela, M., Rampelli, S., Centanni, M., Consolandi, C., Basaglia, G., Turroni, S., Biagi, E., Peano, C., Severgnini, M. (2014): Gut microbiome of the Hadza hunter-gatherers. - Nature communications 5: 3654.

[56] Sekirov, I., Russell, S. L., Antunes, L. C. M., Finlay, B. B. (2010): Gut microbiota in health and disease. - Physiological reviews 90: 859-904.

[57] Shanahan, F. (2002): The host-microbe interface within the gut. - Best practice \& Research Clinical gastroenterology 16(6): 915-931.

[58] Sokol, H., Leducq, V., Aschard, H., Pham, H.-P., Jegou, S., Landman, C., Cohen, D., Liguori, G., Bourrier, A., Nion-Larmurier, I. (2017): Fungal microbiota dysbiosis in IBD. - Gut 66: 1039-1048.

[59] Sonnenburg, J. L., Angenent, L. T., Gordon, J. I. (2004): Getting a grip on things: how do communities of bacterial symbionts become established in our intestine? - Nature immunology 5: 569 .

[60] Sood, A., Amre, D., Midha, V., Sharma, S., Sood, N., Thara, A., Bansal, M., Juyal, G., Thelma, B. K., Seidman, E. (2014): Low hygiene and exposure to infections may be 
associated with increased risk for ulcerative colitis in a North Indian population. - Annals of gastroenterology: quarterly publication of the Hellenic Society of Gastroenterology 27: 219.

[61] Soon, S., Molodecky, N. A., Rabi, D. M., Ghali, W. A., Barkema, H. W., Kaplan, G. G. (2012): The relationship between urban environment and the inflammatory bowel diseases: a systematic review and meta-analysis. - BMC Gastroenterology 12: 51.

[62] Talham, G. L., Jiang, H.-Q., Bos, N. A., Cebra, J. J. (1999): Segmented filamentous bacteria are potent stimuli of a physiologically normal state of the murine gut mucosal immune system. - Infection and immunity 67: 1992-2000.

[63] Umesaki, Y., Okada, Y., Matsumoto, S., Imaoka, A., Setoyama, H. (1995): Segmented filamentous bacteria are indigenous intestinal bacteria that activate intraepithelial lymphocytes and induce MHC class II molecules and fucosyl asialo GM1 glycolipids on the small intestinal epithelial cells in the ex-germ-free mouse. - Microbiology and immunology 39: 555-562.

[64] Walker, A. W., Sanderson, J. D., Churcher, C., Parkes, G. C., Hudspith, B. N., Rayment, N., Brostoff, J., Parkhill, J., Dougan, G., Petrovska, L. (2011): High-throughput clone library analysis of the mucosa-associated microbiota reveals dysbiosis and differences between inflamed and non-inflamed regions of the intestine in inflammatory bowel disease. - BMC Microbiology 11: 7.

[65] Willing, B. P., Dicksved, J., Halfvarson, J., Andersson, A. F., Lucio, M., Zheng, Z., Järnerot, G., Tysk, C., Jansson, J. K., Engstrand, L. (2010): A pyrosequencing study in twins shows that gastrointestinal microbial profiles vary with inflammatory bowel disease phenotypes. - Gastroenterology 139: 1844-1854.

[66] Wu, W., Chen, F., Liu, Z., Cong, Y. (2016): Microbiota-specific Th17 cells: Yin and Yang in regulation of inflammatory bowel disease. - Inflammatory bowel diseases 22: 1473-1482.

[67] Yatsunenko, T., Rey, F. E., Manary, M. J., Trehan, I., Dominguez-Bello, M. G., Contreras, M., Magris, M., Hidalgo, G., Baldassano, R. N., Anokhin, A. P. (2012): Human gut microbiome viewed across age and geography. - Nature 486: 222. 